\title{
Alcohol Drinking Patterns and Contributing Factors among Medical Students, a Systematic Review
}

\author{
Sheehama J A ${ }^{1,3}$ Mbangula $\mathbf{H ~ J}^{2,3}$ Lukolo L $\mathbf{N}^{1}$ \\ ${ }^{1}$ UNAM School of Medicine, South Africa. \\ ${ }^{2}$ Ministry of Health and Social services, South Africa. \\ ${ }^{3}$ TESA Namibia Research, South Africa.
}

Corresponding Author: Sheehama J A, UNAM School of Medicine, South Africa.

Received date: June 24, 2021; Accepted date: December 15, 2021; Published date: January 03, 2022

Citation: Sheehama J A., Mbangula H J., Lukolo L N (2022) Alcohol Drinking Patterns and Contributing Factors among Medical Students, a Systematic Review. J, Clinical Medical Reviews and Reports. 4(1); DOI:10.31579/2690-8794/094

Copyright: (c) 2022, Sheehama J A, This is an open access article distributed under the Creative Commons Attribution License, which permits unrestricted use, distribution, and reproduction in any medium, provided the original work is properly cited.

\section{Abstract}

Background: The use of Alcohol has become an important public health concern with a variety of negative consequences, it is important to understand the variables that may be risk factors for this phenomenon. Further, university students represent a group of individuals who have unique drinking patterns and different risk factors and concerns related to problematic drinking than the population in general.

Legal substances like alcohol accounts for the vast majority of negative medical, economic, and social impact. Although alcohol use occurs across many age groups, young adults aged 18-24 years show the highest rates of alcohol use and have the greatest percentage of problems drinkers (Kandel \& Logan, 1984). Namibia is ranked fifth on the African continent in terms of annual alcohol consumption with the average Namibian consuming 9.62 liters of alcohol per year (WHO 2011). This review addresses problematic drinking and the variables associated with it for medical students. The purpose of this systematic review is to compare the perception and attitude of alcohol consumption among medical students and weigh the factors associated with drinking habits.

Methods: A qualitative and quantitative systematic review of article from multiple search engines. Five articles were within the inclusion criteria thus appraised and reviewed for this paper. The common study method used was cross sectional, with varying sample sizes. Commonly, the use of self-assessment questionnaires and objective AUDIT C and CAGE score evaluation were used frequently between these articles.

Results: The review showed that there are multiple factors that influence the use of alcohol among medical students. Personal factors such as a new found sense of independence, peer pressure, inability to handle academic stressors. Socioeconomic factors include high tolerance of alcohol use in the communities and monthly expenses. It was also noted that the use of alcohol in medical students is higher than the average university student. A highlighted noted is that the use of alcohol is much higher among male than female medical students.

Conclusion: Findings suggested that the perceptions of alcohol use is depended on multiple factors majority being academic perceived stress. It is also noted that continuation of these maladaptive coping mechanisms may lead to dysfunction in the future. The findings of systematic review are limited by the number of articles appraised and reliant on the information provided by the authors.

Keywords: alcohol; students; academic; stress

\section{Introduction}

Alcohol abuse has continued to pose a significant challenges in the world. A study conducted by the Namibian Ministry of health and social services and the international federation of blue cross in partnership with The Finnish institute for health and welfare revealed that $38 \%$ of young adults with a median age of $17-20$ years old have developed tendencies to consume alcohol frequently and in large qualities. This also happens to be the approximate age range students are admitted to University.

Medical students are more prone to alcohol abuse than their peers not attending medical school, especially if they are young, single and under a 
high debt load. (Academic Medicine, 2012). Which leads to the pending question whether Burnout factors such as emotional exhaustion and perceived stress were associated with alcohol dependence among the medical students?

In particular, 'University students are at risk for substance abuse behaviors because of changes in lifestyle, reduced parental support and stress" (M. Davoren, 2016).

According to (Davis and Brantley,2004) ways to cope include adaptive strategies, i.e. problem solving and positive re-examination of a problem, as well as maladaptive coping strategies that reduce the emotional response in the short term but lead to greater difficulties in the long term. As an example, and considering the relationship to alcohol, use of alcohol to reduce an emotional response or to reduce awareness of stressful circumstances is considered to be a maladaptive coping strategy.

Alcoholism is perhaps the most common, devastating, and costly problems in the world (Camy, 2014). Various studies done in different countries have indicated that one group most vulnerable to alcohol abuse is young adults between the ages of 18 and 29, especially college-age young adults. About $25 \%$ of people aged 18 or older reported engaging in binge drinking (five or more drinks in $2 \mathrm{~h}$ for men and four or more drinks in $2 \mathrm{~h}$ for women) in the past month ( $\mathrm{NIH}, 2014)$.

In addition to experiencing these negative alcohol-related consequences, a significant number of heavy-drinking university students also experience more clinically significant problems. While most university students "mature out" of heavy drinking once they leave tertiary education, a considerable number continue to drink heavily and experience more clinically significant problems into adulthood (Maggs 2004).

\section{Methods}

Various search engines were used to collect the data. The selection was based on the comparison of different parameters that are known to influence the use of alcohol selected independently from Google scholar, the BMJ, UNAM library and PubMed. A total of 9647 articles were identified. Upon removal of duplicates, exclusion of articles based on the content and screening of the abstracts, 11 articles were eligible for review, on further scrutiny only 5 articles had met the inclusion criteria and thus included in this review.

Inclusion criteria:

- Articles written in English

- Qualitative and quantitative methodology

- The studies should emphasize on prevalence of alcohol use and risk factors associated

\section{Exclusion criteria}

- The use of other substances (cocaine, marijuana etc)

\section{Study abstraction}

A large scale literature search was conducted independently by one researcher (and overseen thoroughly by the supervisors) to collect studies for inclusion in this review. The data used was extracted from PubMed, Google Scholar, University of Namibia Library and The British Journal of Medicine. Multiple keyword combinations were used: [prevalence OR pattern] AND [drinking OR alcohol OR substances] AND [medical students OR college students]. Over 70000 articles were originally identified using these search criteria.

\section{Data extraction}

Data from the studies were extracted independently by one researcher and over seen by the supervisors for this paper, any disagreements were carefully evaluated and the agreed upon made the bulk of the final data. Extracted data included: Main author, publication year, research design and methodology used, outcomes and main findings.

\section{Results}

A total of 60 articles were identified for possible analysis, however only 5 met the inclusion criteria. The results are presented below:

- $78 \%$ (3777/4847) of medical students reported drinking in the past month, and a third (1668/4847) drank excessively.

- Among excessive drinkers, about three quarters of men and women drank on 14 or fewer days in the past month. On the days excessive drinkers consumed alcohol, 62\% (696/1124) of men drank three or more drinks and 89\% (478/539) of women drank two or more drinks

- The prevalence of alcohol use among students in rural area was higher than that in urban area.

- $\quad$ A study revealed the reason why adolescents from rural area were at higher risk of alcohol use was likely that peer attitudes regarding alcohol use are influenced by lower levels of parental supervision that's less disapproving of adolescent alcohol use and added fact of higher tolerance for alcohol use in rural communities

- A high proportion of students are at risk for alcohol abuse according to the CAGE questionnaire.

- The results of this study suggest that although the quantity of alcohol consumed is not a substantial concern at this time, students might be at risk for future alcohol abuse.

- The prevalence of alcohol use among medical students is high, even though they understand the injuries it may cause.

- Factors such as increase independence, reduced parental supervision and frequent social contact predispose the use and abuse of alcohol.

- Alcohol use among medical students in the US is an ongoing problem.

- Consequences of alcohol use in this sample of medical students included but were not limited to interpersonal altercations, serious suicidal ideation, cognitive deficits, compromised academic performance, and driving under the influence of substances male medical students tend to consume significantly more alcohol than their female peers.

\section{Discussion}

A research indicates that no matter which countries they are from and what cultures they have, males are more likely to drink alcohol. In this study, the findings were that there is no significant urban-rural difference in the prevalence of alcohol use among males. The findings of this systematic review showed that medical students are affected by the same social burdens that weigh down the youth. Contrary to them being the future advocates for healthy living lifestyles in their communities, they are most susceptible due to their lengthy working hours and extensive training years. As opposed to other university faculties, the field of medicine requires a minimum of 4-6 years of training. This factor exposes the students to a magnitude of environmental factors that lead to the greater risk of heavy, frequent alcohol abuse. In addition, students may be kept within the field of study due to multiple reasons some of which are poor academic performance that may lead to repetition of the academic year and lack of funds that may potentiate a cycle of dropping out and returning back to university as high debts are frequently associated with the medical discipline, this is important to note as it causes emotional distress among students and predisposing them to alcohol abuse. Several articles state that alcohol consumption increased for students living on campus, living in a dormitory with a higher number of room-mates. The issue of peer pressure plays a major role in this regard, as the maintenance 
of friendships is considered respectable among young adults. Most articles support the notion that factors such as increased independence, reduced parental supervision, and more social contacts potentially contribute to the increased consumption of alcohol which make the students vulnerable to alcohol abuse.

\section{Conclusion}

The articles revealed that alcohol drinking patterns among medical students are due to multiple factors, of which Academic related factors are the most consistent. The perceived stress endured by medical students during their lengthy years of training highly predispose students to alcohol abuse. The dysfunctional use of alcohol is particularly seen mostly among male then female students and often associated with hazardous intoxicated behaviors. Although most students "grow out" of this phase, it may persistent into adulthood and beyond the years of training causing significant personal and socioeconomic impairment in the future.

\section{Recommendation}

Following the appraisal of these articles it important to identify the main factors related to the consumption of alcohol and formulate solutions to minimize them. The common understanding with in these articles is the perceived stress related drinking that occurs commonly among medical students. This can be curbed by having social workers/counselors on campus that actively help with issues faced by medical students on a daily basis, personally and academically. Furthermore a review of the curriculum to tailor it into a student friendly working environment, working hours and exam time table. Consideration should be put in allowing medical students to live in dormitory rooms as individuals as sharing personal space tends to breed disruptive behaviors among students. University policies should be reinforced strictly to restrain the use of alcohol and other substances on campus grounds and students should be held accountable for these actions. Finally, open conversations should begin at each individual home and at a community level about the societal burdens alcohol and other substances have on the youth, this creates awareness of a growing problem that will affect the future of medicine.

\section{References}

1. A.L. Goldstein, N.P. Barnett, C.T. Pedlow, J.G. Murphy (2007) Drinking in conjunction with sexual experiences among at-risk college student drinkers. Journal of Studies on Alcohol and Drugs, 68 (5) pp. 697-705

2. CAMY (2014) Center of alcohol marketing and youth, Johns Hopkins University, Bloomberg School of Public Health

3. M.L. Cooper, M.R. Frone, M. Russell, P. Mudaret al., (1995) Drinking to regulate positive and negative emotions: A motivational model of alcohol use. Journal of Personality and Social Psychology, 69 (5) pp. 990-1005

4. E.M. Edwards, T. Heeren, D. Rosenbloom, (2009) Age of drinking onset and injuries, motor vehicle crashes, and physical fights after drinking and when not drinking. Alcoholism: Clinical and Experimental Research, 33 (5) pp. 783-790

5. E. Kuntsche, R. Knibbe, G. Gmel, R. Engels (2006) who drinks and why? A review of socio-demographic, personality, and contextual issues behind the drinking motives in young people. Addictive Behaviors, 31 (10), pp. 1844-1857

6. Hingson et al., (2002) Magnitude of alcohol-related mortality and morbidity among US college students ages 18-24 Journal of Studies on Alcohol and Drugs, 63 (2) pp. 136-156

7. R.W. Hingson, W. Zha, E.R. Weitzman (2009) Magnitude of and trends in alcohol-related mortality and morbidity among US college students ages 18-24. Journal of Studies on Alcohol and Drugs, 16 pp. 12-32

8. S.H. Stewart, S.B. Zeitlin, S.B. Samoluk (1996) Examination of a three-dimensional drinking motives questionnaire in a young adult university student sample. Behavioral Research Theories, 34 (1) pp. 61-71

9. H. Wechsler, J.E. Lee, M. Kuo, M. Seibring, T.F. Nelson, H. Lee (2002) Trends in college binge drinking during a period of increased prevention efforts: Findings from 4 Harvard School of Public Health College alcohol study surveys: 1993-2001.Journal of American College Health, 50 (5) pp. 203-217

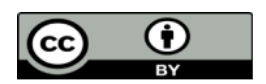

This work is licensed under Creative Commons Attribution 4.0 License
To Submit Your Article Click Here: $\quad$ Submit Manuscript

DOI: $10.31579 / 2690-8794 / 094$
Ready to submit your research? Choose Auctores and benefit from:

$>$ fast, convenient online submission

$>$ rigorous peer review by experienced research in your field

$>$ rapid publication on acceptance

$>$ authors retain copyrights

$>$ unique DOI for all articles

$>$ immediate, unrestricted online access

At Auctores, research is always in progress.

Learn more https://auctoresonline.org/journals/clinical-medical-reviews-andreports- 